\title{
Formation of unsaturated regions in the porous wick of a capillary evaporator
}

\author{
M. Nishikawara ${ }^{1}$, H. Nagano ${ }^{1}$, L. Mottet $^{2}$ and M. Prat ${ }^{2}$ \\ ${ }^{1}$ Department of Aerospace Engineering, Nagoya University, Furo, Chikusa, Nagoya, 464-8603, Japan \\ ${ }^{2}$ INPT, UPS, IMFT (Institut de Mécanique des Fluides de Toulouse), Université de Toulouse, Avenue Camille Soula, F- \\ 31400 Toulouse, France and CNRS; IMFT, F-31400 Toulouse, France \\ *Corresponding author \\ Phone $\quad:+81-52-789-4405$ \\ Email : : nishikawara@ $\quad$ prop2.nuae.nagoya-u.ac.jp \\ Address :381, Building 2 of Graduate School of Engineering, Department of Aerospace Engineering, \\ Nagoya University, Furo-cho, Chikusa-ku, Nagoya, Aichi 464-8603, Japan
}

\begin{abstract}
The heat and mass transfer in a porous wick within a capillary evaporator were analyzed in three dimensions, using a pore network model to simulate immiscible liquid-vapor flow and phase changes in the capillary structure. Characteristics of the porous structure, such as pore radius distribution, permeability and porosity were obtained from measurements of an actual wick and were included in the calculations. Scenarios involving both a fully liquid-saturated and an unsaturated wick containing liquid and vapor were examined under steady state conditions. The location at which the initial vapor phase was generated was identified based on classical nucleation theory. The transition from a saturated to an unsaturated wick was assessed by modeling the distribution of the liquid-vapor interface, and the wicks were compared based on color 3D renderings of temperature and pressure distributions and meniscus curvatures. The maximum temperature of the unsaturated wick exceeded that of the saturated wick although the area of the liquid-vapor interface in the unsaturated wick was five times that in the saturated wick. Since a distribution of pore radii was considered in these calculations, the interface within the wick was not smooth but exhibited asperity. The distribution of meniscus curvatures in the unsaturated wick was much wider compared with the saturated wick. The results obtained in the case of an unsaturated wick demonstrated the occurrence of the heat pipe effect, induced by a distribution of capillary pressures.
\end{abstract}

\section{Keywords}

-Phase Heat Transfer 


\begin{tabular}{|c|c|}
\hline$\underline{A}$ & $\underline{\operatorname{area}\left(\mathrm{m}^{2}\right)}$ \\
\hline$\underline{B}$ & Bond number \\
\hline $\bar{C} a$ & capillary number \\
\hline$\underline{c_{p}}$ & specific heat at constant pressure $(\mathrm{J} / \mathrm{kg} \cdot \mathrm{K})$ \\
\hline$g$ & gravity $\left(\mathrm{m} / \mathrm{s}^{2}\right)$ \\
\hline$g_{\text {th }}$ & flow conductance $\left(\mathrm{m}^{3}\right)$ \\
\hline$\underline{h}$ & heat-transfer coefficient $\left(\mathrm{W} / \mathrm{m}^{2} \cdot \mathrm{K}\right)$ \\
\hline$\underline{H}_{f g}$ & latent heat $(\mathrm{J} / \mathrm{kg})$ \\
\hline$\underline{k}$ & thermal conductivity $(\mathrm{W} / \mathrm{m} \cdot \mathrm{K})$ \\
\hline$\underline{L}$ & length $(\mathrm{m})$ \\
\hline$\dot{m}$ & $\underline{\text { mass flow rate }(\mathrm{kg} / \mathrm{s})}$ \\
\hline$\underline{P}$ & pressure $(\mathrm{Pa})$ \\
\hline$\underline{P e}$ & $\underline{\text { Peclet number }}$ \\
\hline$\dot{Q}_{\text {evap }}$ & heat transferred by evaporation (W) \\
\hline$\dot{Q}_{\text {leak }}$ & heat leak to the compensation chamber (W) \\
\hline$\dot{Q}_{\text {sens }}$ & sensible heat transferred from the inlet to the outlet of the wick (W) \\
\hline$\dot{q}_{a p p l y}$ & heat flux applied to the case $\left(\mathrm{W} / \mathrm{m}^{2}\right)$ \\
\hline$\underline{\text { rpore }}$ & pore radius $(\mathrm{m})$ \\
\hline$\underline{r t h}$ & $\underline{\text { throat radius }(\mathrm{m})}$ \\
\hline$\underline{T}$ & temperature $\left({ }^{\circ} \mathrm{C}\right)$ \\
\hline$\underline{u}$ & velocity $(\mathrm{m} / \mathrm{s})$ \\
\hline
\end{tabular}

\section{Greek}

$\begin{array}{ll}\underline{\varepsilon} & \text { porosity } \\ \underline{\theta}_{\text {cont }} & \text { contact angle (rad) } \\ \underline{\mu} & \underline{\text { viscosity }(\mathrm{Pa} \cdot \mathrm{s})} \\ \underline{v} & \text { kinetic viscosity }\left(\mathrm{m}^{2} / \mathrm{s}\right) \\ \underline{\rho} & \text { density }\left(\mathrm{kg} / \mathrm{m}^{3}\right) \\ \underline{\sigma} & \text { surface tension }\left(\mathrm{N} / \mathrm{m}^{2}\right)\end{array}$

$\underline{\text { Subscripts }}$

$\begin{array}{ll}\underline{\text { cap }} & \underline{\text { capillary }} \\ \underline{\text { cc }} & \underline{\text { compensation chamber }} \\ \underline{\text { cont }} & \underline{\text { contact }} \\ \underline{\text { e }} & \underline{\text { evaporator }} \\ \underline{\text { evap }} & \underline{\text { evaporation }} \\ \underline{\text { eff }} & \underline{\text { effective }} \\ \text { gr } & \text { groove } \\ \underline{\text { int }} & \underline{\text { interface }} \\ \underline{\underline{\text { max }}} & \underline{\text { liquid }} \\ \underline{\text { maximun }} \\ \underline{\underline{\text { nuc }}} & \underline{\text { meniscus }} \\ & \underline{\text { nucleation }}\end{array}$




\begin{tabular}{|c|c|}
\hline sens & $\underline{\text { sensible }}$ \\
\hline sat & saturation \\
\hline sup & superheat \\
\hline & vapor \\
\hline
\end{tabular}

\section{INTRODUCTION}

Loop heat pipes (LHPs) and capillary pumped loops (CPLs) are widely applied to various thermal control devices. They allow significant heat transfer without electric power by taking advantage of capillary pressure in a porous medium and the phase change of a working fluid between liquid and vapor. Unlike conventional heat pipes, the wick in these units is restricted solely to the evaporator, and so longer transport lengths and larger radiation areas are possible.

The capillary evaporators in LHPs and CPLs are normally different from those found in conventional heat pipes. The directions of heat absorption flow and supplied liquid are as in Figure 1(a), while the positioning of heat pipes are the same as in normal evaporators. The capillary evaporator consists of a wick, grooves, casing, and core. Liquid in the wick evaporates due to heating of the casing, and the vapor flows to the grooves due to capillary pressure at the liquid-vapor interface. The liquid efficiently transports heat through condensation and then returns to the evaporator. LHPs and CPLs both operate based on this cycle, and detailed descriptions of these units are available in Ref. [1].

This work focused on heat and mass transfer within the capillary evaporator, since these are complex and determine the performance of the system. Previous analytical studies in Refs. [2-15] identified two main phenomena in the steady-state; a "saturated" behavior in which the wick is fully filled with liquid at a lower heat flux, as in Refs. [2-5], and an "unsaturated" behavior in which the wick exhibits a two-phase state such that both liquid and vapor are present at higher heat flux values, as in Refs. [6-13]. Refs. [2, 3 and 6] developed the first numerical models for the investigation of heat and mass transfer in a capillary evaporator. Ref. [14] analyzed the unsaturated wick using two steady-state solutions: the continuum model and pore network simulation, and examined the influence of pore size distribution, heat flux, and wick thermal conductivity on the liquid-vapor interface and on the thermal performance. Ref. [8] developed a numerical model for an LHP evaporator in the case of either a saturated or unsaturated wick, and concluded that initiation of boiling below the liquid-vapor interface is very unlikely. 3D heat transfer analyses using a continuum approach have also been presented in Refs. [4, 5 and 13]. However, to the best of our knowledge, no model considering the distribution of the porous microstructure in an unsaturated wick in three dimensions has yet been developed.

In the present work, a 3D numerical model simulating heat and mass transfer in the capillary evaporator of saturated and unsaturated wicks was developed, based on a pore network model (PNM), to study immiscible two-phase flow through a porous media and steady-state phase changes. Transition from a saturated wick to an unsaturated wick is also considered, and phase distributions based on capillarity at the liquid-vapor interface are calculated. 


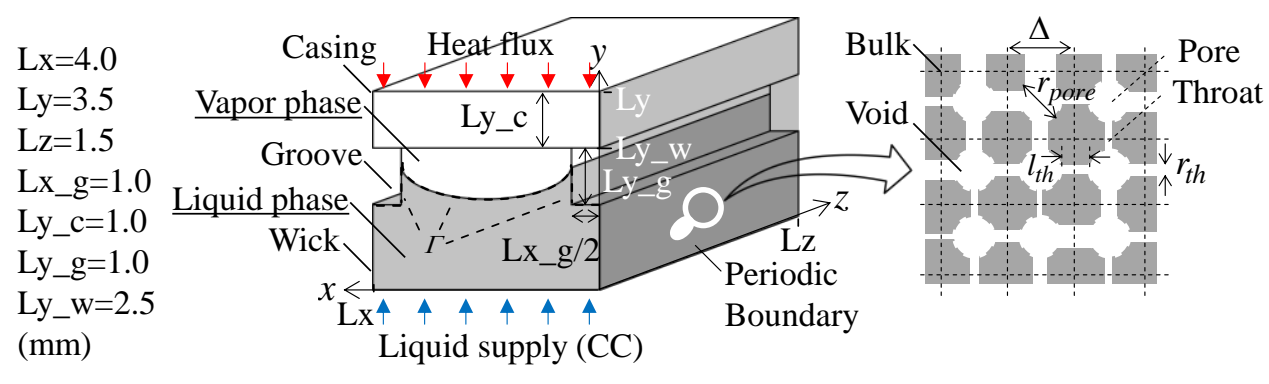

(a)

(b)

FIG. 1. Schematics of (a) the capillary evaporator and (b) the pore network model applied during fluid analysis in the wick.

\section{THREE-DIMENSIONAL NUMERICAL MODEL}

The computational domain is shown in Figure 1(a), corresponding to a portion of the periodic structure of a capillary evaporator. Unlike the half width portions applied in previous studies such as Refs. [2-6, 8-10 and 14], the full width was used, such that the center of the domain is the center of the porous medium, to analyze the asymmetrical characteristics of fluid behavior. The geometry of the capillary evaporator is the same as in Ref. [16]. In this model, a shorter Lz value of $1.5 \mathrm{~mm}$ was applied so as to decrease calculation costs and the casing is included to investigate heat flux distribution at the casing-wick contact surface.

Figure 1(b) shows the pore network model (PNM) for fluid simulation. The PNM divides the voids of the porous structure into spherical pores and cylindrical throats. Unlike the continuum approach used for solving flows in porous media in Refs. [2-4, 6, 8, 9, 11 and 13], the PNM allows a distribution of pore radius values and corresponding local variables. Furthermore, it differs from the lattice Boltzmann method for solving the flow field directly over the entire void in Refs. [17 and 18]. Since local voids are represented as simple shapes associated with throats and pores in the pore network, the present model allows analysis of a greater quantity of pores. Detailed descriptions of the PNM have been previously published in Refs. [14, 15, 19 and 20].

At a low applied heat flux, the liquid-vapor interface stabilizes at the wick-groove interface, representing a saturated state. When the applied heat flux is high and superheating of the liquid phase near the casing exceeds a certain value, vapor bubbles trapped in the porous structure of the wick or in the casing cavity can grow and a vapor phase is created and invades the porous wick. The liquid-vapor interface is stabilized within the wick, representing an unsaturated state.

The main assumptions of the model are: (1) the groove is saturated with a constant temperature and pressure over its entirety, (2) there is a local thermal equilibrium between the bulk solid and fluid in the wick, (3) the fluid is incompressible, (4) the temperature dependence of the fluid properties is considered, (5) the liquid-vapor interface has no thickness, (6) the process is steady-state, and (7) the effects of gravity and thermal radiation are neglected.

\subsection{Governing Equations for Thermal Hydraulics}

In the PNM, the mass flow rate, $\dot{m}_{i j}$, in the throat between pores $\mathrm{i}$ and $\mathrm{j}$ is: 
$\dot{m}_{i j}=\frac{g_{i j}}{v_{-i}} \Delta P_{i j}$

where $g_{i j}$ is the flow resistance in the throat, $v_{-} \mathrm{i}$ is the kinematic viscosity coefficient of phase $I$ (liquid or vapor), and $\Delta P_{i j}$ is the pressure difference between pores $\mathrm{i}$ and $\mathrm{j}$. Assuming Poiseuille flow in a circular pipe, the flow resistance is:

$g_{i j}=\frac{\pi r_{t h}^{4}}{8 l_{t h}}$

where $r_{t h}$ and $l_{t h}$ are the radius and length of the throat. A system of linear equations for the pressure field is obtained by considering mass conservation at the $i$ pore:

$$
\begin{aligned}
& \sum_{j} \dot{m}_{i j}=0, \text { then } \\
& \sum_{j} \frac{g_{i j}}{v_{-} i} \Delta P_{i j}=0 .
\end{aligned}
$$

The energy conservation of the wick, including a convection term and the heat conduction equation of the evaporator casing, is expressed as:

$$
\begin{aligned}
& c_{p_{-} i} \nabla\left(\dot{m}_{i j} T\right)=k_{\text {eff_i }} A \nabla^{2} T, \quad \text { and } \\
& k_{\text {case }} A \nabla^{2} T=0
\end{aligned}
$$

where $c_{p}$ is the specific heat at constant pressure of the fluid, $T$ is the temperature, $A$ is the cross-sectional area of the control volume, $k_{\text {case }}$ is the thermal conductivity of the casing, and $k_{\text {eff }}$ is the effective thermal conductivity of the wick. This is obtained by volume averaging the thermal conductivity of the bulk solid and the working fluid with porosity $\varepsilon$, as in Eq. (7).

$$
k_{\text {eff_i }}=\varepsilon k_{-i}+(1-\varepsilon) k_{b u l k}
$$

In a saturated wick, the liquid evaporates over the entire wick-grooves interface while, in an unsaturated wick, the evaporation interface consists of a part of the wick-grooves interface and the liquid-vapor interface in the wick. The evaporation interface is defined as $\Gamma$, as in Figure 1(a). Note that condensation is also possible at the $\Gamma$ interface. The $\Gamma$ interfacial conditions are expressed as follows.

The mass continuity condition is as below:

$\dot{m}_{n_{-} l}=\dot{m}_{n_{-} v}$.

The energy conservation condition at the wick-grooves interface is given by the following equations: 
$-\left.k_{e f f \_l} A \frac{\partial T}{\partial n}\right|_{\Gamma}=h_{i} A\left(T-T_{g r}\right)$,

$h_{i} A\left(T-T_{g r}\right)=\dot{m}_{n} H_{f g}$.

The energy conservation condition and the temperature condition assuming thermal equilibrium at the $\Gamma$ interface in the wick are:

$\left[-\left.k_{e f f_{-} v} A \frac{\partial T}{\partial n}\right|_{\Gamma}\right]-\left[-\left.k_{e f f_{-} l} A \frac{\partial T}{\partial n}\right|_{\Gamma}\right]=\dot{m}_{n} H_{f g}, \quad$ and

$T_{v}=T_{l}=T_{s a t}$,

where $n$ is the direction normal to the $\Gamma$ interface, $h_{i}$ is the interfacial heat-transfer coefficient calculated using the equation in Ref. [21], $T_{g r}$ is the temperature of the grooves, $H_{f g}$ is the latent heat of the working fluid, and $T_{\text {sat }}$ is the saturation temperature of the working fluid, calculated from the pressure based on the saturation curve of the P-T diagram. The region in the grooves is saturated and its temperature is determined from the pressure in that area: $P_{g r} . P_{g r}$ is, in turn, calculated from the pressure of the compensation chamber (CC), $P_{c c}$, and the pressure loss from the grooves to the CC, $\Delta P_{\text {lines, }}$, as follows:

$P_{g r}=P_{c c}+\Delta P_{\text {lines }}$,

where $P_{c c}$ is calculated from the temperature of the CC, assumed to be constant, and $\Delta P_{\text {lines }}$ is calculated from the mass flow rate in the loop, assuming Poiseuille flow in a circular pipe, as presented in Ref. [22].

The other boundary conditions, commonly applied in previous studies, are: (1) uniform heat flux at the heating surface $(y=L y)$,

$\dot{q}_{a p p l y}=\left(-k_{\text {case }} \frac{\partial T}{\partial y}\right)_{y=L y}$,

(2) thermal connection via a contact heat-transfer coefficient of $10,000 \mathrm{~W} / \mathrm{m}^{2} \mathrm{~K}$, as applied in Ref. [23], at the casing-wick contact surface ( $y=$ Ly_w), (3) a convective boundary at the bottom of the wick $(y=$ 0 ) with a heat-transfer coefficient of $100 \mathrm{~W} / \mathrm{m}^{2} \mathrm{~K}$, as used in a previous study [13], (4) constant pressure values of $P_{c c}$ and $P_{g r}$ at the bottom of the wick $(\mathrm{y}=0)$ and the wick-grooves interface in instances when the $\Gamma$ interface is not included,

$P_{y=0}=P_{c c}$ and

$P_{\text {wick-groove }}=P_{g r}$,

(5) impervious walls at $\mathrm{z}=0, \mathrm{Lz}$, and $\mathrm{y}=\mathrm{Ly} \_\mathrm{w}$,

$\left.\dot{m}_{z}\right|_{z=0, L z}=0$ and 
$\left.\dot{m}_{y}\right|_{y=L y_{-} w}=0$,

(6) adiabatic conditions at $\mathrm{z}=0$ and at $\mathrm{Lz}$, the casing-groove interface, and the wick-grooves interface in instances when the $\Gamma$ interface is not included,

$P_{y=0}=P_{c c}$ and

$P_{\text {wick-groove }}=P_{g r}$,

and (7) periodic boundaries at $\mathrm{x}=0$ and $\mathrm{Lx}$

$T_{x=0}=T_{x=L x+\Delta}$ and

$P_{x=0}=P_{x=L x+\Delta}$.

\subsection{Characteristics of the porous structure}

The parameters characterizing the porous microstructure are the pore radius, $r_{p o r e}$, the throat radius, $r_{t h}$, and the length of the throat, $l_{t h}$, as in Figure 1(b). These variables may be tuned to accurately set the porosity, permeability and pore radius distribution. In this work, a polytetrafluoroethylene (PTFE) wick was employed, as in Ref. [16], with porosity and permeability values of 0.34 and $2.0 \times 10^{-14} \mathrm{~m}^{2}$, respectively. The mode of the pore radii and the maximum pore radius were 1.2 and $2.1 \mu \mathrm{m}$. The throat radii were constructed using a probability density function with a lognormal distribution to fit the pore radius distribution as measured by mercury porosimetry as in Ref. [24]. The permeability of the resulting wick was calculated using computational permeability, as in Ref. [15]. All of the main porous characteristics were fit to the measured values. Porous characteristics were determined as in Ref. [25].

\subsection{Algorithm for the transition from a saturated to an unsaturated wick and invasion of the vapor phase}

The method applied to determine the solutions of the above equations is summarized in the flow chart in Fig. 2. In this process, there is one convergence associated with the calculations for the saturated wick and two convergences in the case of the unsaturated wick. In the algorithm for the saturated wick, Eqs. (5) and (6) are first solved with no mass flow rate, using standard finite volume methods with cubic lattices incorporating a mesh size, $\Delta$, of $0.1 \mathrm{~mm}$ and a total of 105,472 nodes. From the results, the mass flow rate at the $\Gamma$ interface is calculated, which is imposed as the boundary condition for Eq. (4). After the pressure field is solved with the same mesh size of the finite volume method, the mass flow rates in the wick and the overall LHP system, as well as the pressure drop from the grooves to the CC, are calculated. Finally, the newly calculated values for the pressure drop from the grooves to the $\mathrm{CC}$ and the mass flow rates in the wick are compared with the previous values to ascertain the degree of convergence of the thermal hydraulics. Eqs. (5) and (6) and then solved again with the updated mass flow rates. The above calculation loop is iterated until the results are seen to converge.

The energy balance of the overall system is expressed as: 
$\dot{Q}_{\text {leak }}+\dot{Q}_{\text {evap }}+\dot{Q}_{\text {sens }}=\int \dot{q}_{\text {apply }} d A$

where $\dot{Q}_{\text {leak }}$ is the heat leak to the core, $\dot{Q}_{\text {evap }}$ is the heat transferred by phase change, and $\dot{Q}_{\text {sens }}$ is the sensible heat transferred from the inlet to the outlet of the wick. The right side of this equation represents the total amount of applied heat. Each term in the left side is calculated as follows:

$$
\begin{aligned}
& \dot{Q}_{\text {leak }}=\int\left(-\left.k_{e f f-l} l \frac{d T}{d x}\right|_{y=0}\right) d A \\
& \dot{Q}_{\text {evap }}=\sum_{\Gamma} \dot{m}_{n} H_{f g}, \text { and } \\
& \dot{Q}_{\text {sens }}=\left\lfloor\sum_{\Gamma} \dot{m} c_{p_{-} l} T-\sum_{y=0} \dot{m} c_{p_{-} l} T\right\rfloor+\left\lfloor\sum_{g r-\text { wick }} \dot{m} c_{p_{-}} T-\sum_{\Gamma} \dot{m} c_{p_{-} v} T\right\rfloor .
\end{aligned}
$$

In this work, Eq. (23) was satisfied with a maximum relative error of less than $1 \%$.

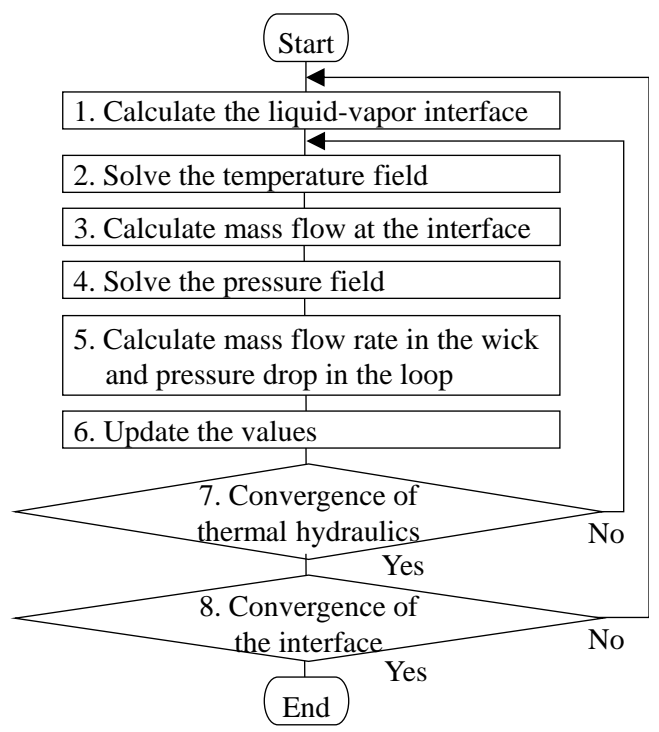

FIG. 2. Flowchart for the numerical solution process. Steps $2-7$ are applicable in the case of a saturated wick and steps $1-8$ in the case of an unsaturated wick.

After the results for the saturated wick were obtained, the transition to an unsaturated wick was considered. This transition occurs because of the growth of bubbles in the wick, and the classical equation for boiling critical superheat, $\Delta T_{\text {nuc }}$, is:

$$
\Delta T_{n u c}=\frac{2 \sigma T_{s a t}}{\rho_{-v} r H_{f g}}
$$


where $\sigma$ is the surface tension, $\rho_{-} v$ is the density of the vapor and $r$ is the radius of the bubbles. When superheating of the liquid phase occurred at the casing-wick contact surface, $\Delta T_{\text {sup }}$ exceeded $\Delta T_{n u c}$ and the calculations shifted to those associated with an unsaturated wick, as in Eq. (28):

$$
\Delta T_{n u c}<\Delta T_{\text {sup }}=T-T_{\text {sat }} .
$$

Eq. (28) can be verified in several liquid phase nodes. The initial vapor phase was generated at the pores corresponding to the maximum value of $\Delta T_{\text {sup }}-\Delta T_{n u c}$. Following vapor phase initiation, the calculations modeled the vapor path connected to the grooves, applying an invasion percolation pattern (IP), as in Refs. [19 and 20], in the first layer of pores in contact with the casing until the vapor connected to the grooves. The throats are invaded one by one in an IP process. The invaded throat has the maximum radius in all throats at the interface. Calculations involving an unsaturated wick began with a phase distribution obtained in the above manner. Following convergence of the thermal hydraulics, convergence of the pressure balance at the liquid-vapor interface was obtained. The possible maximum capillary pressure in a throat is:

$$
P_{c a p_{-} \max }=\frac{2 \sigma \cos \theta}{r_{t h}}
$$

where $\theta$ is the contact angle. When the pressure difference between the vapor and liquid phases at the interface in the throat, $\Delta P_{\text {int }}$, exceeded the maximum capillary pressure in the throat, as in Eq. (30), the vapor phase displaced the liquid phase (equivalent to an invasion process):

$$
P_{c a p_{-} \max }<\Delta P_{\text {int }}=P_{v}-P_{l} \text {. }
$$

Here $P_{v}$ and $P_{l}$ are the vapor and liquid phase pressures at the interface. If the invading menisci are calculated directly using Eq. (30), the number of menisci can be overestimated. According to Ref. [12], less than 13 throats are invaded at the same time. In this model, therefore, the number of moving menisci at any given time was adjusted based on the following simplified algorithm. A coefficient, $c_{\text {int }}$, was introduced such that the condition for the invading meniscus was expressed as follows:

$$
P_{c a p_{-} \max }<c_{\text {int }} \times \Delta P_{\text {int }} \text {. }
$$

The value of this coefficient was raised gradually from a chosen minimum value. Once some menisci were satisfied by the equation, the appropriate throats were invaded, following which the thermal hydraulics were again solved. From the solved pressure field, the invading menisci were again calculated using the chosen minimum $c_{\text {int }}$ value (not last step $c_{\text {int }}$ ) until the coefficient eventually reached a value of unity. At this point, the condition described by Eq. (31) exactly corresponded to Eq. (30) and hence the calculations ceased since there was no further intrusion of menisci. The number of moving menisci at any given time could be finely tuned by the above algorithm and, in these calculations, the minimum coefficient value was set at 0.01 and raised in increments of 0.01 and the average number of moving menisci at any given time was 6.27. It was confirmed that $c_{\text {int }}$ was small enough not to influence the phase distribution at the equilibrium state.

After the interface was invaded, liquid clusters were able to form, representing isolated regions of liquidfilled pores disconnected from the liquid reservoir. These were not stable under steady-state conditions 
because they were not connected to a liquid supply and therefore they disappeared as soon as they were formed. The Hoshen-Kopelman algorithm in Ref. [26] was used to identify and then remove these clusters.

\section{RESULTS AND DISCUSSION}

The calculation conditions were as follows. The heat flux applied to the casing was $1.25 \mathrm{~W} / \mathrm{cm}^{2}$ and this heat flux satisfied the transition conditions of Eq. (28). The compensation chamber (CC) saturation temperature was $29.4^{\circ} \mathrm{C}$. The wick material was PTFE with a bulk thermal conductivity of $0.25 \mathrm{~W} / \mathrm{m} \cdot \mathrm{K}$ and the thermal conductivity of the stainless steel casing was $16 \mathrm{~W} / \mathrm{m} \cdot \mathrm{K}$. The working fluid was ethanol. For these conditions, the order of magnitude of the capillary number, $C a$, the bond number, $B$, and the Peclet number of each phase, $P e_{-} v$ and $P e_{-} l$, were $4.73 \times 10^{-7}, 3.00 \times 10^{-7}, 0.32$, and 0.41 , respectively.

$C a=\frac{u_{l} \mu_{l}}{2 \sigma \cos \theta}=\frac{\left[\dot{q}_{a p p l y} /\left(\rho_{l} H_{f g}\right)\right] \mu_{l}}{2 \sigma \cos \theta}$

$B=\frac{r_{\text {pore_mode }}^{2} \rho_{l} g}{2 \sigma \cos \theta}$

$P e_{v}=\frac{\rho_{v} c_{p_{-} v} u_{v} L y_{-} w}{k_{e f f_{-} v}}=\frac{c_{p_{-} v}\left(\dot{q}_{a p p l y} / H_{f g}\right) L y_{-} w}{k_{e f f_{-} v}}$

$P e_{l}=\frac{\rho_{l} c_{p_{-} l} u_{l} L y_{-} w}{k_{e f f_{-} l}}=\frac{c_{p_{-} l}\left(\dot{q}_{a p p l y} / H_{f g}\right) L y_{-} w}{k_{e f f_{-} l}}$

where, $u$ is cross-sectional average flow velocity at the wick inlet, $\mu_{l}$ is viscosity of liquid, $r_{\text {pore_mode }}$ is mode pore radius, $\rho_{l}$ is liquid density, and $g$ is the acceleration of gravity. Figure 3 shows the temperature fields of the saturated and unsaturated wicks, including the casing, wick, and grooves, in which gray surfaces represent the liquid-vapor interface associated with the phase change. In both fields, the maximum temperature occurred at the upper surface of the casing, and the minimum temperature at the bottom of the wick. The maximum temperature of the unsaturated wick was higher than that of the saturated wick. Figure 4 shows the pressure fields of the saturated and unsaturated wicks excluding the casing. On the unsaturated wick, vapor phase had larger distribution because kinetic viscosity of vapor was larger than that of liquid. In the saturated wick, the maximum pressure occurred in grooves, and in the unsaturated wick, it occurred in the central part of vapor phase of the wick. Generated vapor in the central part was high pressure due to large pressure loss flowing to the grooves. Similar pressure distribution are presented in the previous 2D works [6 and 9]. Figure 5(a) presents the number of moving menisci at each step and the number of menisci at the liquid-vapor interface, starting from the initial generation of vapor to the equilibrium state, in the case of the unsaturated wick. The results for the liquidvapor interface in this plot have been normalized to the interface of the saturated wick. The area of the liquid-vapor interface in the unsaturated wick is five times that in the saturated wick. Although larger area of the interface, the casing temperature for the unsaturated wick was higher than the saturated one. The reason why the heat transfer decreases is occurrence of vapor phase region. Applied heat flux evaporates at the liquid-vapor interface through the vapor phase. Because thermal conductivity of the vapor phase is low, temperature difference between the casing and the interface enlarges depending on the size of the vapor phase. Therefore, both of the size of the vapor phase and the area of the interface is important on the enhancement of the heat transfer in the unsaturated wick. Immediately before the vapor phase reaches 
the opposite position to its initial location (step 102 in Figure 5(a)), the number of moving menisci is at a maximum of 233. Although this value is much larger than the value of 13 reported in Ref. [12], it can still be considered reasonable, taking into account the effects of the larger network size and higher temperature gradient of the wick in the present study. Figures 5(b) and (c) show the interface at the initial step in which the first vapor phase is generated and the final step of the equilibrium state. The location of the first vapor phase is at the triple boundary wick-casing-groove interface, rather than the center of the wick along the $\mathrm{x}$ direction, owing to the inclusion of the saturation temperature and temperature dependence of the fluid properties at each node in Eq. (28). Note that the location of first bubble depends on a working fluid, so, in some case (for example, ammonia), the location is in the center portion of the wick contacting the case. An animated sequence showing the transition of the interface is presented as Figure 6, generated by calculating the interface position at each iteration of the invasion process. As the equilibrium state is approached, isolated liquid clusters are formed and subsequently disappear. Since there is a distribution of pore radii, an irregular interface results, as shown in Figure 5(c). This interface recedes to a greater extent along the center of the $\mathrm{x}$ direction.

Figure 7 shows the distribution of menisci curvatures, $1 / r_{m e n i}$, at the liquid-vapor interface. The curvature at the interface throat was calculated by the Laplace equation given below as Eq. (36).

$$
\frac{1}{r_{m e n i}}=\frac{\Delta P_{\mathrm{int}}}{2 \sigma}
$$

The dimensionless curvature values, using the pore radius mode as a reference, are shown in Figure 7. The curvature distribution represents the distribution of capillary pressures and the saturated wick is seen to have small curvature values overall with a narrow distribution, while the distribution of the unsaturated wick is much wider. In the case of the unsaturated wick, the curvature at the liquid-vapor interface is larger than that at the wick-groove interface. Heat transfer by evaporation and condensation in the wick may occur owing to the flow induced by a distribution of capillary pressures, corresponding to the heat pipe effect, and these results demonstrate that condensation occurs at 517 of the 2538 throats within the liquidvapor interface, although the condensation flow rate is small, equal to only $2.18 \%$ of the total flow rate. A heat pipe effect during the development of vapor pockets was reported in Ref. [12] and the present study indicates that the heat pipe effect appears under steady-state conditions.
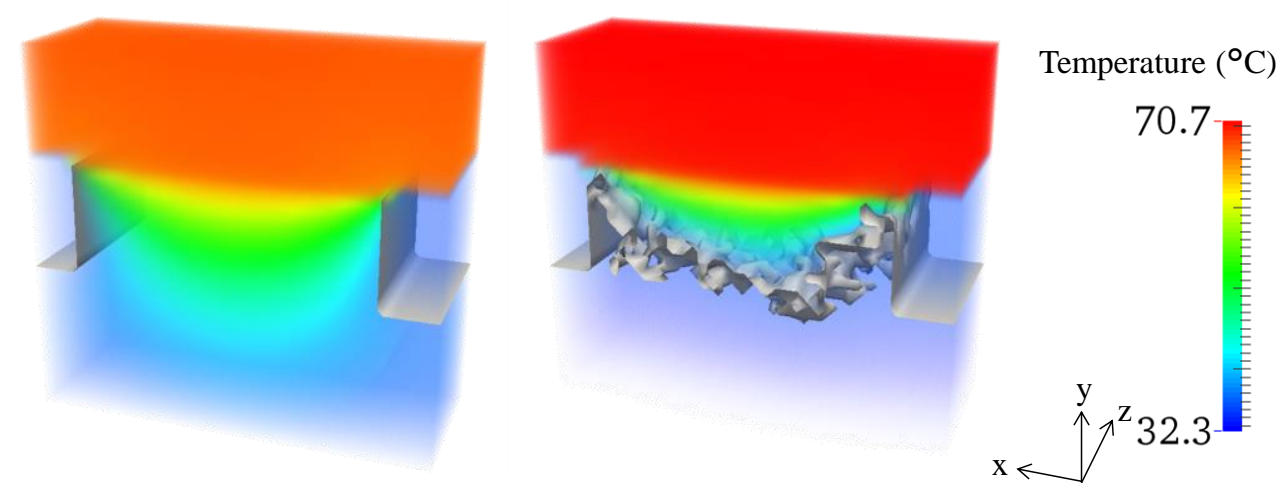

FIG. 3. Temperature fields of the saturated (left) and the unsaturated (right) wick, including the casing and grooves. 

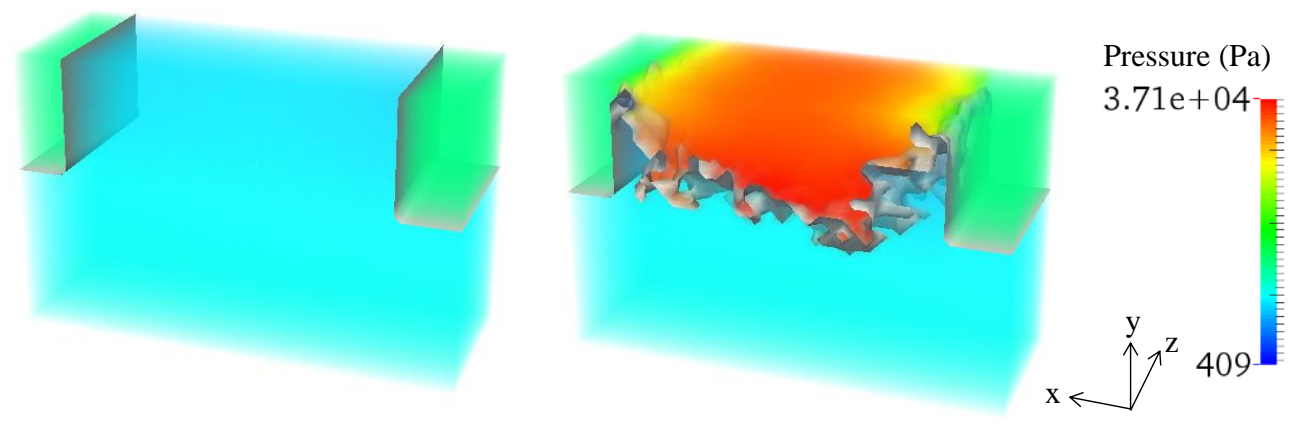

FIG. 4. Pressure fields of the saturated (left) and the unsaturated (right) wick, including grooves.

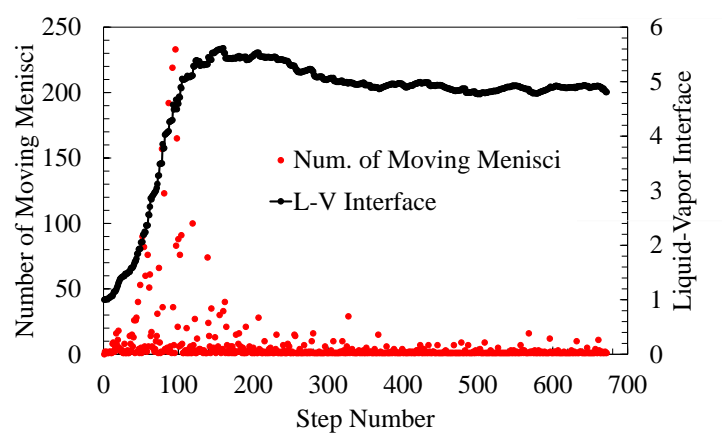

(a)

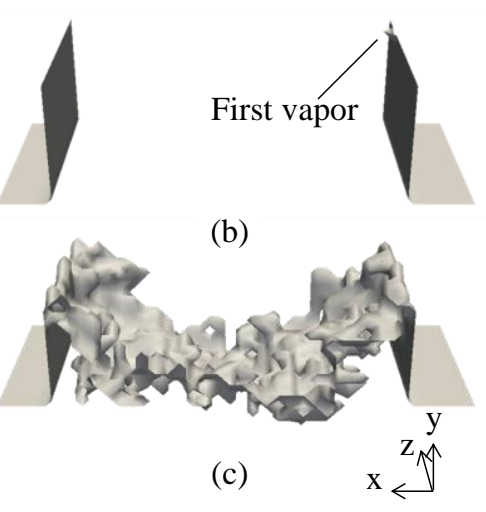

(c)

FIG. 5. (a) Variations in the number of moving menisci and the liquid-vapor interface during the invasion process. The distributions of the interface at (b) the first step and (c) the final step of calculations.
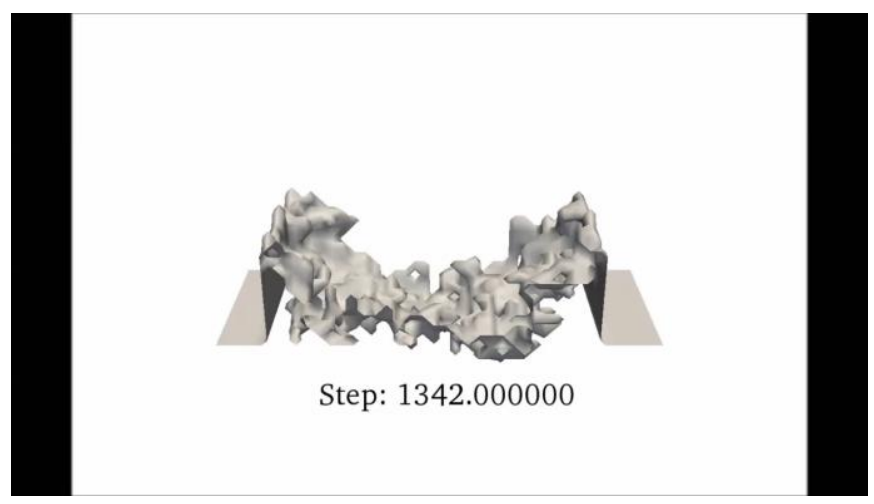

FIG. 6. Animation of the liquid-vapor interface movement during the invasion process. The number of steps in this animation is double the number in Fig. 3(a) since two images before and after the cluster removal procedure have been included. (Video data) 

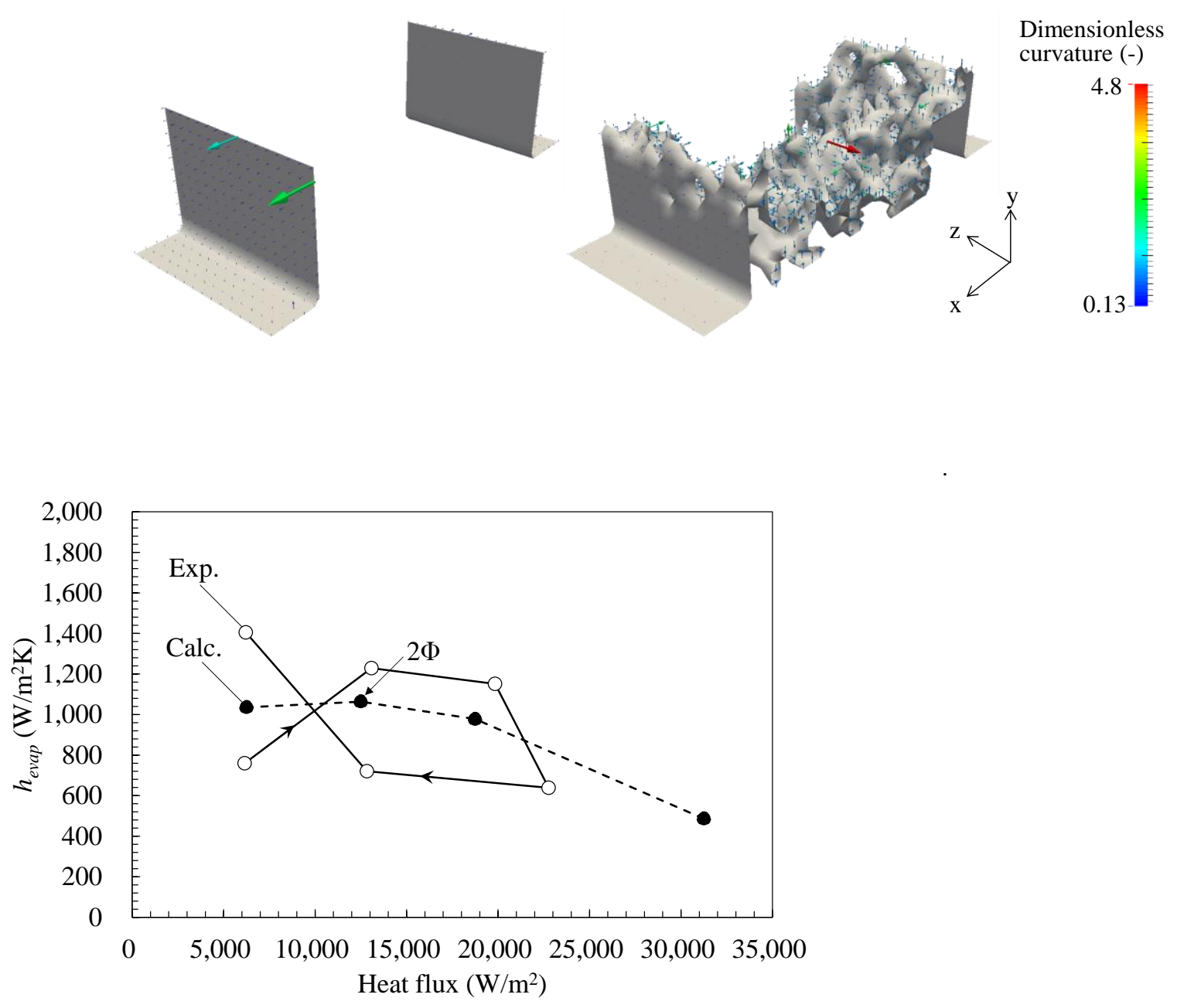

Figure 8 presents the comparison of the evaporator heat-transfer coefficient between the calculation and the experiment. The heat-transfer coefficient were calculated as follows,

$$
h_{\text {evap }}=\frac{\dot{q}_{\text {apply }}}{\left(T_{e_{-} \max }-T_{g r}\right)}
$$

where, $T_{e_{-} \max }$ is the maximum temperature on the casing. The detail of the experiment is presented in Ref. [16]. The wick and working fluid are the same as the calculation. The shape of the evaporator are different between the experiment of cylinder and the calculation of rectangle, but width and height of groove and ratio of contact area between the casing and the wick to the cross-sectional area of the casing are fitted to be similar geometry. In the experiment, heat load increased to $23,000 \mathrm{~W} / \mathrm{m}^{2}$, then decreased to the end. The profile is indicated by arrows in Figure 8. Because the $\mathrm{CC}$ temperature changed at each heat flux in the experiment, to consider temperature dependence of the fluid property, the other elements of the LHP were included in this analysis. Numerical model for the other elements was based on Ref. [22]. When the heat flux applied to the evaporator varied, the saturation temperature in the grooves changed, but the difference was small and less than $2{ }^{\circ} \mathrm{C}$. In the calculation, because the unsaturated wicks at more than $13,000 \mathrm{~W} / \mathrm{m}^{2}$ of heat flux were analyzed from the saturated wick, comparable test data was during increasing of heat load. The difference between the experiment and the calculation were $13 \%$ at 13,000 $\mathrm{W} / \mathrm{m}^{2}$ and $15 \%$ at $19,000-20,000 \mathrm{~W} / \mathrm{m}^{2}$. At the lowest heat flux, because error of measurement make an larger impact than the other heat fluxes, the difference between the experiment and the calculation may be larger. Taking account into hysteresis phenomena is necessary to predict during decreasing of heat load. 
In this model, the superheat for transition to the unsaturated wick is estimated with ideal condition of Eq. (27), but measurement by the experiment is necessary to predict with higher accuracy.

\section{SUMMARY AND CONCLUSIONS}

A 3D numerical model of the heat and mass transfer in a capillary evaporator with either a fully liquidsaturated or a vapor-liquid wick under steady state conditions was developed, and a pore network model was used to simulate immiscible liquid-vapor flow with phase changes in a capillary structure with a distribution of pore radii. The transition from a saturated to an unsaturated wick owing to the formation and growth of bubbles in the wick resulting from boiling of a liquid from critical superheating was considered. The liquid-vapor interface area and number of moving menisci during the transition from saturation to unsaturation were calculated and presented.

Two important results were obtained from the simulations. Firstly, in this calculation using ethanol as a working fluid, the location at which the initial vapor phase was generated was identified as the triple line formed by the wick-groove-casing interface. Secondly, the heat pipe effect induced by a distribution of capillary pressures was demonstrated by the results obtained for the unsaturated wick. Additionally, the differences between the saturated and unsaturated wicks with regard to heat and mass transfer were clarified.

\section{ACKNOWLEDGMENTS}

This research was partly supported by a Grant-in-Aid for JSPS Fellows (No. 25148) and by JST, PRESTO. LM and MP gratefully thank CNES and Airbus Defence and Space for their financial support. A super computer system in the Information Technology center of Nagoya University was used for the calculations.

\section{REFERENCES}

[1] Y. F. Maydanik, Loop heat pipes, Applied Thermal Engineering 25 (5-6) (2005) 635-657. http://dx.doi.org/10.1016/j.applthermaleng.2004.07.010

[2] C. Yiding and A. Faghri, Analytical solutions of flow and heat transfer in a porous structure with partial heating and evaporation on the upper surface, International Journal Heat and Mass Transfer 37 (10) (1994) 1525-1533. http://dx.doi.org/10.1016/0017-9310(94)90154-6

[3] C. Yiding and A. Faghri, Conjugate analysis of a flat-plate type evaporator for capillary pumped loops with three-dimensional vapor flow in the groove, International Journal Heat and Mass Transfer 37 (3) (1994) 401-409. http://dx.doi.org/10.1016/0017-9310(94)90074-4

[4] L. Ji, and G. P. Peterson, 3D heat transfer analysis in a loop heat pipe evaporator with a fully saturated wick, International Journal Heat and Mass Transfer 54 (1-3) (2011) 564-574. http://dx.doi.org/10.1016/j.ijheatmasstransfer.2010.09.014 
[5] Q. Li, K. Zhao, and Y. M. Xuan, Simulation of flow and heat transfer with evaporation in a porous wick of a CPL evaporator on pore scale by lattice Boltzmann method, International Journal Heat and Mass Transfer 54 (13-14) (2011) 2890-2901. http://dx.doi.org/10.1016/j.ijheatmasstransfer.2011.03.010

[6] A. S. Demidov and E. S. Yatsenko, Investigation of heat and mass transfer in the evaporation zone of a heat pipe operating by the 'inverted meniscus' principle, International Journal Heat and Mass Transfer 37 (14) (1994) 2155-2163. http://dx.doi.org/10.1016/0017-9310(94)90317-4

[7] T. S. Zhao and Q. Liao, On capillary-driven flow and phase-change heat transfer in a porous structure heated by a finned surface: measurements and modeling, International Journal Heat and Mass Transfer 43 (7) (2000) 1141-1155. http://dx.doi.org/10.1016/S0017-9310(99)00206-9

[8] T. Kaya and J. Goldak, Numerical analysis of heat and mass transfer in the capillary structure of a loop heat pipe, International Journal Heat and Mass Transfer 49 (17-18) (2006) 3211-3220. http://dx.doi.org/10.1016/j.ijheatmasstransfer.2006.01.028

[9] C. Ren, Q. S. Wu, and M. B. Hu, Heat transfer with flow and evaporation in loop heat pipe's wick at low or moderate heat fluxes, International Journal Heat and Mass Transfer 50 (11-12) (2007) 22962308. http://dx.doi.org/10.1016/j.ijheatmasstransfer.2006.10.029

[10] M. A. Chernysheva and Y. F. Maydanik, Heat and Mass Transfer in Evaporator of Loop Heat Pipe, Journal of Thermophysics and Heat Transfer 23 (4) (2009) 725-731. http://dx.doi.org/10.2514/1.43244

[11] Z. M. Wan, W. Liu, Z. K. Tu, and A. Nakayama, Conjugate numerical analysis of flow and heat transfer with phase change in a miniature flat plate CPL evaporator, International Journal Heat and Mass Transfer 52 (1-2) (2009) 422-430. http://dx.doi.org/10.1016/j.ijheatmasstransfer.2008.06.019

[12] C. Louriou and M. Prat, Pore network study of bubble growth by vaporisation in a porous medium heated laterally, International Journal of Thermal Sciences 52 (2012) 8-21. http://dx.doi.org/10.1016/j.ijthermalsci.2011.09.008

[13] M. A. Chernysheva and Y. F. Maydanik, 3D-model for heat and mass transfer simulation in flat evaporator of copper-water loop heat pipe, Applied Thermal Engineering 33-34 (2012) 124-134. http://dx.doi.org/10.1016/j.applthermaleng.2011.09.025

[14] C. Figus, Y. Le Bray, S. Bories, and M. Prat, Heat and mass transfer with phase change in a porous structure partially heated: continuum model and pore network simulations, International Journal Heat and Mass Transfer 42 (14) (1999) 2557-2569. http://dx.doi.org/10.1016/S0017-9310(98)00342-1

[15] M. Prat, Pore network models for the study of transfers in the porous wick of loop heat pipes, Heat Pipe Science and Technology, An International Journal 1 (2) (2010) 129-149. 
http://dx.doi.org/10.1615/HeatPipeSciTech.v1.i2.20

[16] M. Nishikawara and H. Nagano, Parametric experiments on a miniature loop heat pipe with PTFE wicks, International Journal of Thermal Sciences 85 (2014) 29-39. http://dx.doi.org/10.1016/j.ijthermalsci.2014.05.016

[17] N. S. Martys and H. Chen, Simulation of multicomponent fluids in complex three-dimensional geometries by the lattice Boltzmann method, Physical Review E 53 (1) (1996) 743-750.

http://dx.doi.org/10.1103/PhysRevE.53.743

[18] A. K. Gunstensen and D. H. Rothman, Lattice-boltzmann studies of immiscible two-phase flow through porous media, Journal of Geophysical Research 98 (B4) (1993) 6431-6441.

http://dx.doi.org/10.1029/92JB02660

[19] M. Prat, Recent advances in pore-scale models for drying of porous media, Chemical Engineering Journal 86 (1-2) 153-164 (2002).

http://dx.doi.org/10.1016/S1385-8947(01)00283-2

[20] M. Prat and F. Bouleux, Drying of capillary porous media with a stabilized front in two dimensions, Physical Review E 60 (5), 5647-5656 (1999).

http://dx.doi.org/10.1103/PhysRevE.60.5647

[21] V.P. Carey, Liquid-Vapor Phase-Change Phenomena, first ed., Taylor \& Francis, 1992, p. 112.

[22] M. Nishikawara, H. Nagano, and T. Kaya, Transient Thermo-Fluid Modeling of Loop Heat Pipes and Experimental Validation, Journal of Thermophysics and Heat Transfer 27 (4) 641-647 (2013). http://dx.doi.org/10.2514/1.T3888

[23] T. Kaya, R. Pérez, C. Gregori, and A. Torres, Numerical simulation of transient operation of loop heat pipes, Applied Thermal Engineering. 28 (8-9) 967-974 (2008).

http://dx.doi.org/10.1016/j.applthermaleng.2007.06.037

[24] T. Allen, Particle Size Measurement, Surface Area and Pore Size Determination, Fifth ed., Chapman and Hall, New York, 1997, p.149.

[25] M. Nishikawara, H. Nagano, and M. Prat, Evaporator heat-transfer analysis of a loop heat pipe with low thermal conductivity wicks, Proceedings of the 17th International heat pipe conference, Kanpur, India, 13-17 October 2013.

[26] J. Hoshen and R. Kopelman, Percolation and cluster distribution. I. Cluster multiple labeling technique and critical concentration algorithm, Physical Review. B 14 (8), 3438-3445 (1976). http://dx.doi.org/10.1103/PhysRevB.14.3438 